\title{
Fabrication of Positively Charged Fluorescent Polymer Nanoparticles for Cell Imaging and Gene Delivery
}

\author{
Lin Wei, ${ }^{1}$ Di Zhang, ${ }^{2}$ Xuanfang Zheng, ${ }^{1}$ Xuyao Zeng, ${ }^{2}$ Youlin Zeng, ${ }^{1}$ Xinbo Shi, ${ }^{3}$ Xin Su, ${ }^{4}$ and Lehui Xiao ${ }^{\varpi, 2}$ \\ 1. Key Laboratory of Chemical Biology \& Traditional Chinese Medicine Research, Ministry of Education, Key Laboratory of Phytochemical R\&D of Hunan \\ Province, College of Chemistry and Chemical Engineering, Hunan Normal University, Changsha, Hunan, 410081, China; \\ 2. State Key Laboratory of Medicinal Chemical Biology, Tianjin Key Laboratory of Biosensing and Molecular Recognition, College of Chemistry, Nankai \\ University, Tianjin, 300071, China; \\ 3. Hunan Provincial Key Laboratory of Food Science and Biotechnology, College of Food Science and Technology, Hunan Agricultural University, Changsha, \\ 410128, China \\ 4. Beijing Key Laboratory of Bioprocess, College of Life Science and Technology, Beijing University of Chemical Technology, Beijing, 100029, China. \\ W. L., D. Z. and X. Z. contributed equally to this work. \\ $\triangle$ Corresponding author: Email: lehuixiao@nankai.edu.cn; Fax: +86-022-23500201
}

(C) Ivyspring International Publisher. This is an open access article distributed under the terms of the Creative Commons Attribution (CC BY-NC) license (https://creativecommons.org/licenses/by-nc/4.0/). See http://ivyspring.com/terms for full terms and conditions.

Received: 2017.09.25; Accepted: 2017.11.28; Published: 2018.02.15

\begin{abstract}
Development of efficient non-viral gene delivery vector has aroused great attention in the past few decades. In this study, we reported a new gene delivery vector, positively charged fluorescent conjugated polymer nanoparticles (CPNPs), for efficient gene transfection and in-situ intracellular fluorescence imaging. The microscopic and spectroscopic characterizations demonstrated that these CPNPs possess decent fluorescence performance (e.g. with fluorescence quantum yield of $70.7 \pm 0.3 \%$ ) and small size dimension of $\sim 3.6 \pm 0.3 \mathrm{~nm}$ (DLS result). Fast and efficient cellular translocation capability was observed according to the time-dependent living cell imaging experiments. Nearly all of the cells were loaded with CPNPs after co-incubation for $2 \mathrm{~h}$ regardless of the cell type. In comparison with the commonly used gene delivery vector, lipofectamine 2000 (with gene transfection efficiency of $55 \pm 5 \%$ for pEGFP), the gene expression efficiency with the positively charged CPNPs (70 $\pm 3 \%$ for pEGFP) was improved significantly. Intracellular fluorescence imaging results demonstrated that the CPNPs could actively assemble close to the periphery of nuclei. Disassembly was not observed even $36 \mathrm{~h}$ later, which greatly facilitates releasing of pDNA close to the periphery of nuclei and thus promotes the gene transfection efficiency.
\end{abstract}

Key words: Conjugated polymer nanoparticles; Gene delivery; Single particle imaging; Living cell imaging; Fluorescence microscopy.

\section{Introduction}

Gene therapy, a technique using foreign nucleic acid (such as plasmid, minivector DNA or siRNA) as medicine to repair defective genes, has shown great potential to treat human diseases that occur from defective genes like cystic fibrosis, macular degeneration, Parkinson's disease, and different types of cancers [1-4]. The development of efficient gene therapy strategies has thus aroused great attention during the past few decades [5-7]. Viral vectors such as adenoviruses and retroviruses are commonly used in gene therapy owing to the high efficiency of cellular translocation as well as the non-selectivity toward cellular membrane $[5,8]$. Although most of the viral vectors are uniquely suited for gene transfer as they can penetrate cell membranes via direct fusion or endocytosis, their routine use in both regular research laboratories and clinical settings are greatly limited due to safety concerns such as undesirable immune response, risk of tumorigenesis and insertional mutagenesis [8]. As a consequence, exploring nonviral gene delivery materials has continuously received considerable attention because these materials can be structurally varied, are relatively safe, and have an ability to carry large and diverse genetic materials into cells.

Until now, a number of studies involving 
non-viral gene delivery systems, such as cationic lipids having one or two hydrophobic tails, polycations, dendrimers and histones, were carried out [9-16]. Results demonstrate that non-viral gene delivery would be a potential approach for the treatment of genetic diseases. For example, enhanced gene delivery and siRNA silencing by gold nanostructures coated with positively charged molecules were readily achieved in either in vitro or in vivo studies [17-19]. Peng et al. also demonstrated that amphiphilic poly(-amidoamine) PAMAM dendrimers could be served as effective vectors for siRNA delivery in diverse disease models [20,21].

However, some studies have shown that changes in protein expression following transfection via non-viral materials can be highly variable. The variability in protein expression is believed to reflect endosomal sequestration which lead to subsequent nucleic acid damage and thus reduced levels of exogenous protein expression [22-26].

Recent studies have demonstrated that association of nucleic acid to the nanoparticle surface (i.e. gold and silica) could result in improvement in protein yields which has been hypothesized to be due to reduced endosomal degradation of the nucleic acid $[27,28]$. Generally, as an efficient gene delivery vector, three major trafficking barriers need to be overcome: (1) the passage of DNA across the plasma membrane, (2) protection and release of DNA molecules, and (3) the passage of DNA cross the nuclear membrane. On this basis, the effectiveness of gene transfection is difficult to study without visualizing the exact transport noninvasively. Exploring intracellular movement may offer deep insight into the precise mechanism of non-viral gene delivery. Therefore, there is a urgent need to develop sensitive and noninvasive methods that could be performed to overcome the challenges of gene therapy such as utilizing nano-dimensional materials to carry genes across cellular membrane barriers and exploiting unique optical (or magnetic) properties for noninvasive and spatiotemporal molecular imaging.

In this work, we demonstrated a novel nanoparticle, small size and positively charged fluorescent conjugated polymer nanoparticle (CPNP), for the efficient gene delivery as well as real-time fluorescence imaging. Through nanoprecipitation of fluorescent conjugated polymer (i.e. PFBT) with zwitterionic lipid molecules (propylene glycol amine cationic lipid) together, positively charged CPNPs were obtained with $\xi$ potential of $+46.8 \mathrm{mv}$. This positively charged nanoparticle exhibits greatly improved fluorescence quantum yield and small size dimension. Fast and universal cellular translocation capability was observed according the comprehensive cellular uptake experiments. $100 \%$ cellular loading efficiency was observed after co-incubation of the nanoparticle with the cell for $2 \mathrm{~h}$. Furthermore, in comparison with the typically adopted gene delivery vector, lipofectamine 2000 (with gene transfection efficiency of $55 \pm 5 \%$ for pEGFP), the gene expression efficiency was improved significantly $(70 \pm 3 \%$ for pEGFP). Due to the attractive optical and biological performances of these CPNPs, they may find broad applications for nucleic acid delivery in future applications of therapeutic genes or RNAs.

\section{Materials and Methods}

\section{Chemicals and Materials}

pEGFP (enhanced green fluorescent protein plasmid) and pDsRed (red fluorescence protein plasmid) were gifted from Prof. Dr. Shuanglin Xiang (Hunan Normal University, college of life science). Propylene glycol amine cationic lipids with different length of carbon tails (R12, R14, R16 and R18) were gifted from Prof. Dr. Youlin Zeng (Hunan Normal University, Chinese patent: \#201110444269.5). The chemical structure of propylene glycol amine cationic lipid and PFBT are show in Figure S1. Lipofectamine 2000 was obtained from Invitrogen Ltd. (Paisley, UK). Poly [(9,9-dioctylfluorenyl-2,7-diyl)-alt-co-(1,4-benzo$\left(2,1^{\prime}, 3\right)$-thiadiazole)] (PFBT) was purchased from American Dye Source, Inc. (Quebec, Canada). Dimethyl sulfoxide (DMSO), 3-(4,5-dimethylthiazol-2yl)-2,5-diphenyltetrazolium bromide (MTT), anhydrous tetrahydrofuran (THF) and other chemicals not mentioned were purchased from Sigma-Aldrich (St. Louis, Mo, USA).

\section{Fabrication of Positively Charged Polymer Nanoparticles}

The CPNPs were synthesized based on the nanoprecipitation method [29,30]. Typically, $10 \mu \mathrm{L}$ of $\mathrm{R} 16(10 \mathrm{mg} / \mathrm{mL}$ in THF) and $10 \mu \mathrm{L}$ of PFBT $(1 \mathrm{mg} / \mathrm{mL}$ in THF) were added to $1 \mathrm{~mL}$ of THF solution in a vial. This mixture was then quickly injected into $6 \mathrm{~mL}$ of deionized water under vigorous sonication at room temperature. THF was then removed by blowing the sample with nitrogen and heated at $85{ }^{\circ} \mathrm{C}$ for $2 \mathrm{~h}$. After the reaction was completed, the solution was gradually cooled down to room temperature and then filtered through a $0.22 \mu \mathrm{m}$ filter to remove aggregates. The aqueous solution was further purified by ultrafiltration (ultrafiltration tube, MWCO: $10 \mathrm{kDa}$ ) three times at $7500 \mathrm{rpm}$ for $10 \mathrm{~min}$ to remove the excess chemicals in the solution and then concentrated to $1.5 \mathrm{~mL}$ by centrifugation. The solution was stored at $4{ }^{\circ} \mathrm{C}$ before use. 


\section{Cell Culture and Cellular Uptake Experiments}

Hek 293, Mat, MCF-7, PC-3 and HeLa cells were purchased from American Type Culture Collection (ATCC, Manassas, VA, USA). Before the cellular uptake studies, the cells were maintained in Dulbecco's modified Eagle's medium (DMEM, Gibco, Thermo Fisher Scientific Inc.) and supplemented with 10\% fatal bovine serum (Gibco, Thermo Fisher Scientific Inc.) at $37{ }^{\circ} \mathrm{C} / 5 \% \mathrm{CO}_{2}$ in a humidified atmosphere. Cells were seeded at a density of $1 \times 10^{5}$ cells per dish within a $35 \mathrm{~mm}$ culture dish and cultured overnight to reach an appropriate cell density.

For the cellular uptake experiments, $20 \mu \mathrm{L}$ of CPNPs $(100 \mu \mathrm{g} / \mathrm{mL})$ was added to the cell culture dish and co-incubated with the five types cells as noted above for $2 \mathrm{~h}$ at $37^{\circ} \mathrm{C}$. Prior to the fluorescence imaging experiments, the medium was removed, and the cells were washed with PBS buffer twice to remove nonspecifically bound CPNPs on the cell surface.

\section{Gene Delivery with CPNPs}

All of the CPNPs/pDNA (pEGFP and pDsRed) complexes were freshly prepared before gene transfection experiments. By using Hek 293 cell line (a human embryonic kidney cell line) as a gene transfection model, Hek 293 cells were seeded at a density of $4.0 \times 10^{4}$ cells within $35 \mathrm{~mm}$ culture dish and cultured overnight to reach an appropriate cell density. $4 \mu \mathrm{g}$ CPNPs and $0.80 \mu \mathrm{g}$ pDNA (the optimum CPNPs/pDNA ratios according to the gel retardation assay) were mixed together in $100 \mu \mathrm{L}$ OptiMEM medium for $30 \mathrm{~min}$ at room temperature. Then, the complexes were diluted with $1 \mathrm{~mL}$ fresh serum-free DMEM and incubated with the cells for $4 \mathrm{~h}$. After that, $1 \mathrm{~mL}$ of complete culture medium was added to each well and the cells were further cultured for $36 \mathrm{~h}$. pEGFP and pDsRed expressed in those cells were characterized by an inverted fluorescence microscope (Ti-U, Nikon, Japan). As a control, a commercial transfection reagent, Lipofectamine 2000, was used to compare the transfection efficiency with CPNPs. The procedures for gene transfection are the same as the guidelines provided by the company. Typically, $3 \mu \mathrm{L}$ of Lipofectamine 2000 was mixed with $0.8 \mu \mathrm{g}$ plasmid DNA to form the cargo/pDNA complex in $100 \mu \mathrm{L}$ OptiMEM medium for $30 \mathrm{~min}$ at room temperature. The subsequent steps are the same as noted above for CPNPs based transfection experiments.

\section{Results and Discussion}

\section{Fabrication of Positively Charged CPNPs}

Nanoprecipitation, and mini-emulsion are two commonly adopted methods for the fabrication of water-soluble fluorescent CPNPs with hydrophobic conjugated polymer [29-34]. Compared with the mini-emulsion method, nanoprecipitation is a more convenient method. The polymer nanoparticles made by this method typically exhibit good colloidal stability, bright fluorescence, versatile surface chemistry as well as high product yield $[29,34]$. Due to the attractive chemical and optical merits, these polymer nanoparticles have been applied to bio-imaging, single particle tracking as well as chemical sensing. In the present study, we demonstrated a small size, bright as well as positively charged CPNPs for gene delivery based on the nanoprecipitation method, Figure 1a.

An amphiphilic lipid molecule, propylene glycol amine cationic lipid (see the supporting information Figure S1), was utilized as the ligand molecule to synthesize positively charged CPNPs. By changing the length of the carbon tail, the hydrophobicity as well as the fluidity of this compound can be readily modulated. Figure $\mathbf{1 b}$ shows the fluorescence spectra of CPNPs made by cationic lipids with different carbon tail lengths. Extending the length of the carbon tail, the fluorescence intensity of CPNPs increased accordingly, indicative of an improved fluorescence QY. This improved fluorescence performance might be partly owing to the reduced aggregation state inside the polymer core [35]. For regular CPNPs without ligand molecules, the fluorescence $Q Y$ normally reduced drastically after the nanoprecipitation process due to the nearby interchain or intrachain $\Pi$ orbital stacking, which results in non-radiative species across the polymer chain, such as excimers, aggregates, and defects. Reminiscent optical performance improvement after the introduction of multidentate amphiphilic compounds was also observed before [36,37]. It is worth to note that the fluorescence QY of the CPNPs made by this method is greatly improved in contrast to the regular CPNPs. The highest absolute fluorescence QY of the CPNPs reached $70.7 \pm 0.3 \%$ when using the cationic lipid molecule with carbon tail length of 16 . Here we noted the compound as R16. This greatly improved QY is mainly ascribed to the fraction of hydrophobic part, which increases significantly when using small molecules instead of polymeric structure. As a consequence, the state of $\Pi$ orbital stacking could be alleviated, further reducing the rate of non-radiative way. To characterize the surface charge of CPNPs, $\xi$ potential was measured and summarized in Figure 1c. All of CPNPs made by this method exhibit highly positive charge $(>+30 \mathrm{mV})$, indicative of good colloidal stability in aqueous surrounding. 
a)

Positively charge
conjugated polym
nanoparticles

\section{Positively charged conjugated polymer}

Nanoprecipitation
with cationic lipid

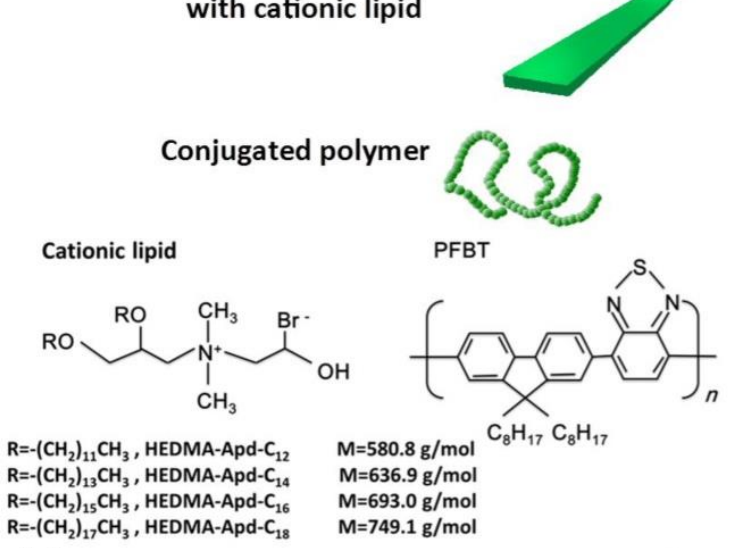

b)

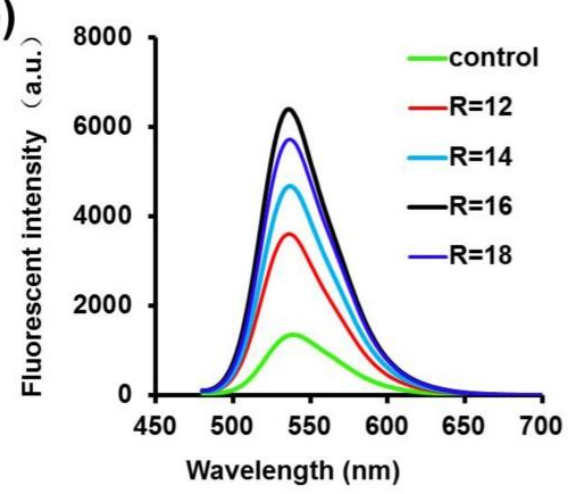

c)
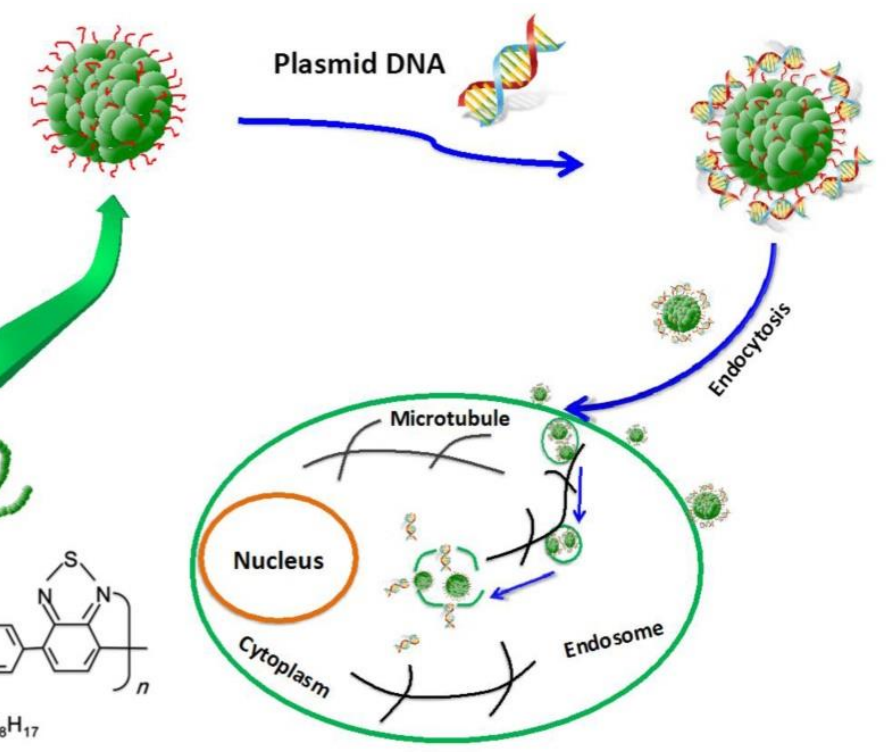

\begin{tabular}{|c|c|c|c|}
\hline & DLS Size (nm) & $\xi$ potential (mv) & QY (\%) \\
\hline Control & $23.6 \pm 0.6$ & $-28.0 \pm 1.2$ & $36.1 \pm 1.0 \%$ \\
\hline $\mathrm{R}=12$ & $6.8 \pm 0.4$ & $36.3 \pm 0.8$ & $57.6 \pm 0.6 \%$ \\
\hline $\mathrm{R}=14$ & $4.2 \pm 0.3$ & $36.4 \pm 1.6$ & $63.4 \pm 0.5 \%$ \\
\hline $\mathrm{R}=16$ & $3.6 \pm 0.3$ & $46.8 \pm 0.9$ & $70.7 \pm 0.3 \%$ \\
\hline $\mathrm{R}=18$ & $3.7 \pm 0.2$ & $37.9 \pm 1.1$ & $65.8 \pm 0.8 \%$ \\
\hline
\end{tabular}

Figure 1. a) Schematic illustration of the procedures for positively charged CPNPs fabrication. b) Ligand dependent (with different carbon tail length noted as R12, R14, R16 and R18 respectively) fluorescence emission spectra of positively charged CPNPs. The black line shows the control CPNPs prepared without the ligand molecule, propylene glycol amine cationic lipid. c) The DLS, $\xi$ potential and fluorescence QY of the CPNPs as noted in figure b).

\section{Optical Performance of the Fluorescent CPNPs}

Since CPNPs made by the ligand of R16 exhibit superior QY and strong surface charge, in the following experiments, we focused on this kind of CPNPs and explored its optical performance as well as biological application capability. As demonstrated in Figure 2a, this positively charged CPNPs dispersed separately in the TEM image with diameter around $3 \pm 0.6 \mathrm{~nm}$. The good colloidal stability of these CPNPs is largely due to the superior positive charge on the particle surface, resulting strong electrostatic repulsion force. The microscopic size characterization is in good accordance with the ensemble spectroscopic result, DLS measurement $(\sim 3.6 \pm 0.3 \mathrm{~nm})$, Figure $\mathbf{2 b}$. To monitor the gene delivery process, the brightness and fluorescence stability against photoblinking of the vector are two essential prerequisites for fluorescence-based imaging. A typical epi-fluorescence image of these CPNPs on the cover glass surface is shown in Figure 2c. Individual bright spots with comparable fluorescence intensity is observed with a laser power of $2 \mathrm{~mW}$ and exposure time of $20 \mathrm{~ms}$. Under this imaging condition, the averaged signal-to-noise ratio of this CPNPs reached 20, which is satisfactory for single particle tracking application. Furthermore, the fluorescence stability of these CPNPs against photoblinking is characterized. Representative fluorescence intensity tracks as a function of time from individual spots on the fluorescence image are shown in Figure 2d. Distinct from other quantum-sized fluorescent materials, i.e. inorganic semiconducting Qdots [38,39], metal clusters [40,41], and fluorescent proteins [42], these small size CPNPs demonstrated good optical stability for single particle imaging, Figure S2.

For biological imaging applications, the $\mathrm{pH}$ as well as salt concentration of the surrounding will be changed greatly in a tempo-spatially dependent 
manner. In this regard, besides the single particle

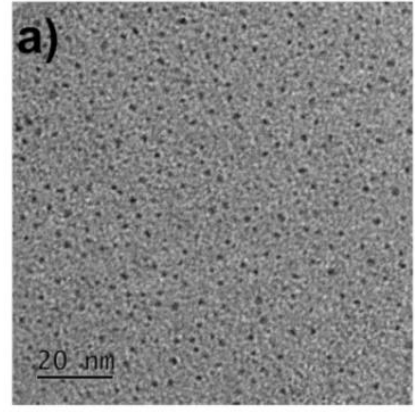

c)

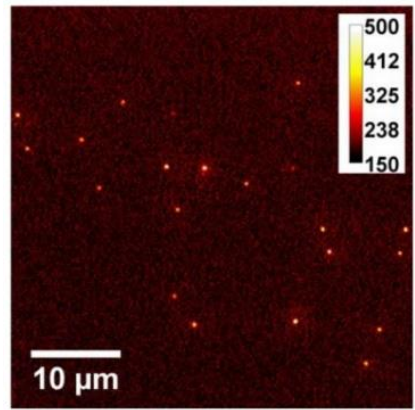

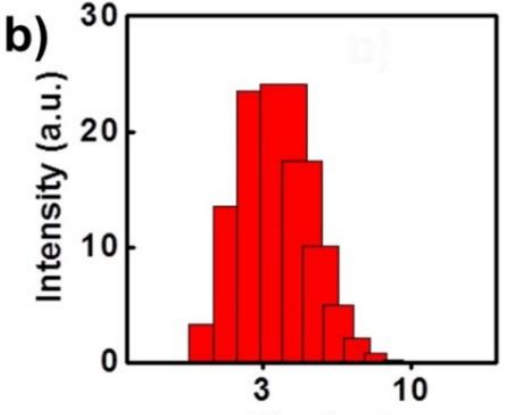

d)

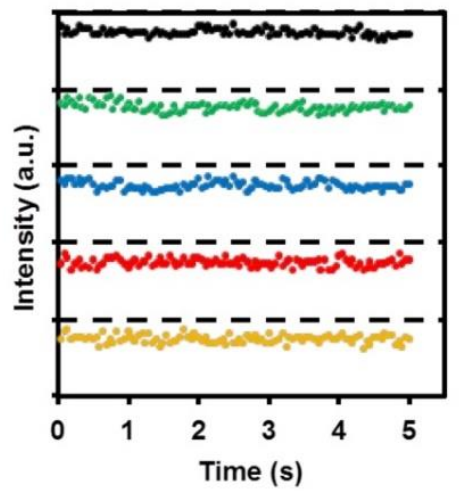

Figure 2. a) TEM image of the CPNPs fabricated with the ligand of propylene glycol amine cationic lipid with the carbon tail length of 16 (R16). b) and c) are the DLS and fluorescence microscopic image of the corresponding CPNPs. d) Representative single particle fluorescence intensity tracks as a function of time in figure $\mathrm{c}$ ).

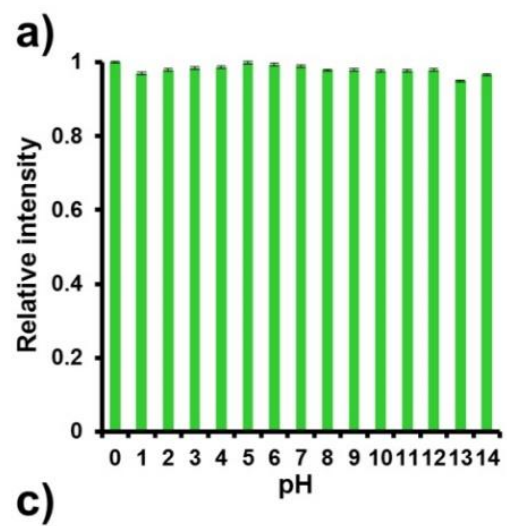

b)
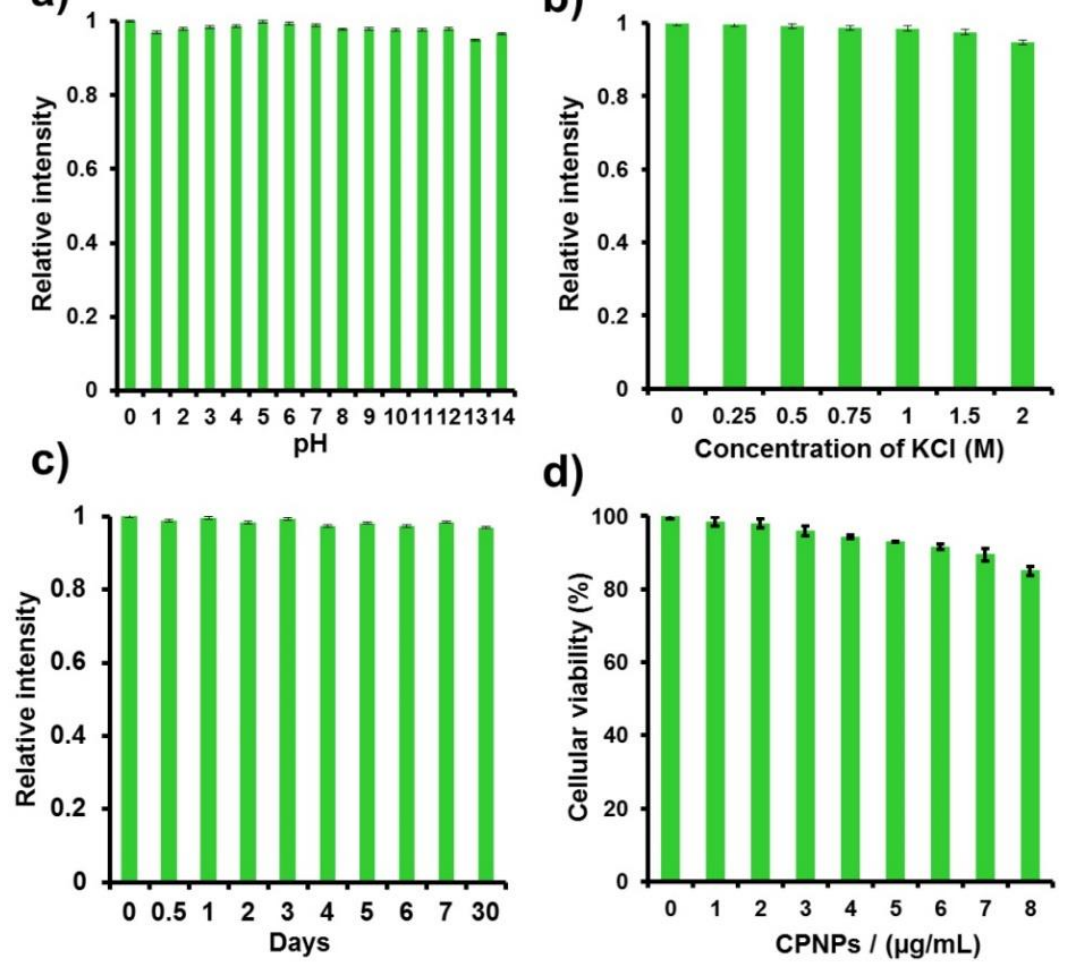

Figure 3. Fluorescence stability characterization of CPNPs made by the ligand of R16. The relative fluorescence intensity of CPNPs, a) under different $\mathrm{pH}$ solutions, $\mathbf{b}$ ) inside $\mathrm{KCl}$ solution with different concentrations, and c) under DI water as a function of time. d) The cytotoxicity of CPNPs (with Hek 293 as the model) as a function of mass concentration evaluated by MTT colorimetric assay. characterizations, we further explored the fluorescence stability of CPNPs against environmental disturbances at ensemble scale, i.e., $\mathrm{pH}$ and salt concentration. Interestingly, the CPNPs exhibit decent fluorescence stability in a broad $\mathrm{pH}$ range, Figure 3a. Even under extremely acid and basic surroundings, the CPNPs still maintained good fluorescence brightness. Similar phenomenon was also observed under high salt solutions. No quenching effect was noted when the salt concentration reached $2 \mathrm{M}$, Figure $3 \mathrm{~b}$. These decent colloidal and optical performances would be greatly ascribed to the compact and hydrophobic core together with the strong surface charge, which play a significant role in shielding the quenching species. Moreover, it is worth to note that these CPNPs could be stored for a long time without noticeable aggregation. The fluorescence intensity of these CPNPs kept constant even for 30 days when stored at $4{ }^{\circ} \mathrm{C}$.

\section{Cellular Cytotoxicity, Translocation Efficiency, and Selectivity of CPNPs}

Cytotoxicity is an important issue that needs to be characterized before the gene transfection study. In this regard, the cytotoxicity of CPNPs as a function of mass concentration was evaluated by MTT colorimetric assay in comparison with a blank control [43,44]. As indicated in Figure 3d, the cell viability is more than $94 \%$ at the concentration of $4.0 \mu \mathrm{g} / \mathrm{mL}$ (the dosage used for the subsequent gene transfection experiments). Further increasing the concentration, slight decrease in cell viability was noted. Even 2 fold increase in the dosage of the sample only resulted in $15 \%$ of cell death, indicating a good biocompatibility. In contrast to lipo2000, CPNPs display improved biocompatibility under the similar mass concentration Figure S3.

Next, it is also essential to understand the cellular translocation efficiency and selectivity of the CPNPs toward different types of cells. Typically, the cellular uptake process for nanoparticles is controlled by some 
factors such as size, shape, surface chemistry and so on, which collectively determine the cellular translocation mechanism [45-47]. For positively charged nanoparticles, the electrostatic association between the negatively charged cell membrane and CPNPs will promote the subsequent cellular internalization but will not guarantee an efficient uptake process. As a gene delivery cargo for the further guiding of DNA toward the nuclei, it is important to elucidate the cellular uptake efficiency. On this account, five kinds of cells were co-cultured with the CPNPs for $2 \mathrm{~h}$ and then imaged with a fluorescence microscope, Figure 4a. Green fluorescence was noted from all of the cells independent of the cell type, indicative of an excellent cellular internalization efficiency. This universal and efficient cellular translocation efficiency might be largely attributed to the small size dimension as well as the positive surface charge of the nanoparticle. These two factors play a synergistic effect on the membrane translocation process, which could be either energy dependent or independent.

To further disclose the detailed cellular uptake mechanism, a control experiment was conducted to co-culture the CPNPs with HeLa cell at $4{ }^{\circ} \mathrm{C}$. Under this condition, the energy-dependent endocytosis pathways, e.g. clathrin and caveolae mediated endocytosis, are almost inhibited. Figure $4 \mathrm{~b}$ and $4 \mathrm{c}$ illustrate the fluorescence images of the cells co-cultured with CPNPs for $2 \mathrm{~h}$ at 37 and $4{ }^{\circ} \mathrm{C}$ respectively. Noticeable difference in the fluorescence intensity between these two sets of images was observed. Obviously, the cellular uptake process is more efficient under $37^{\circ} \mathrm{C}$. Around 10 -fold increase was observed according to the quantitative fluorescence intensity analysis. However, it is worthy of note that, even the energy-dependent endocytosis process was inhibited; few CPNPs can still be observed inside the cell. Besides the temperature control experiment, we also modulated the endocytosis process with different drugs i.e., dynasore (an inhibitor of clathrin-mediated endocytosis by deactivating dynamic-GTPase) and genistein (an inhibitor of caveolae-mediated endocytosis by deactivating isoflavone tyrosine kinase), Figure S4. Under one of the drug treatment, the cellular uptake efficiency decreased in contrast to the results at $37{ }^{\circ} \mathrm{C}$ without drug treatment. No complete inhibition was noted. However, a more efficient inhibition effect was observed when both of these two drugs treated with the cells simultaneously. These results together demonstrated that multiplexed cellular uptake mechanisms involve in the membrane penetration process, i.e., endocytosis, physical penetration and so on.

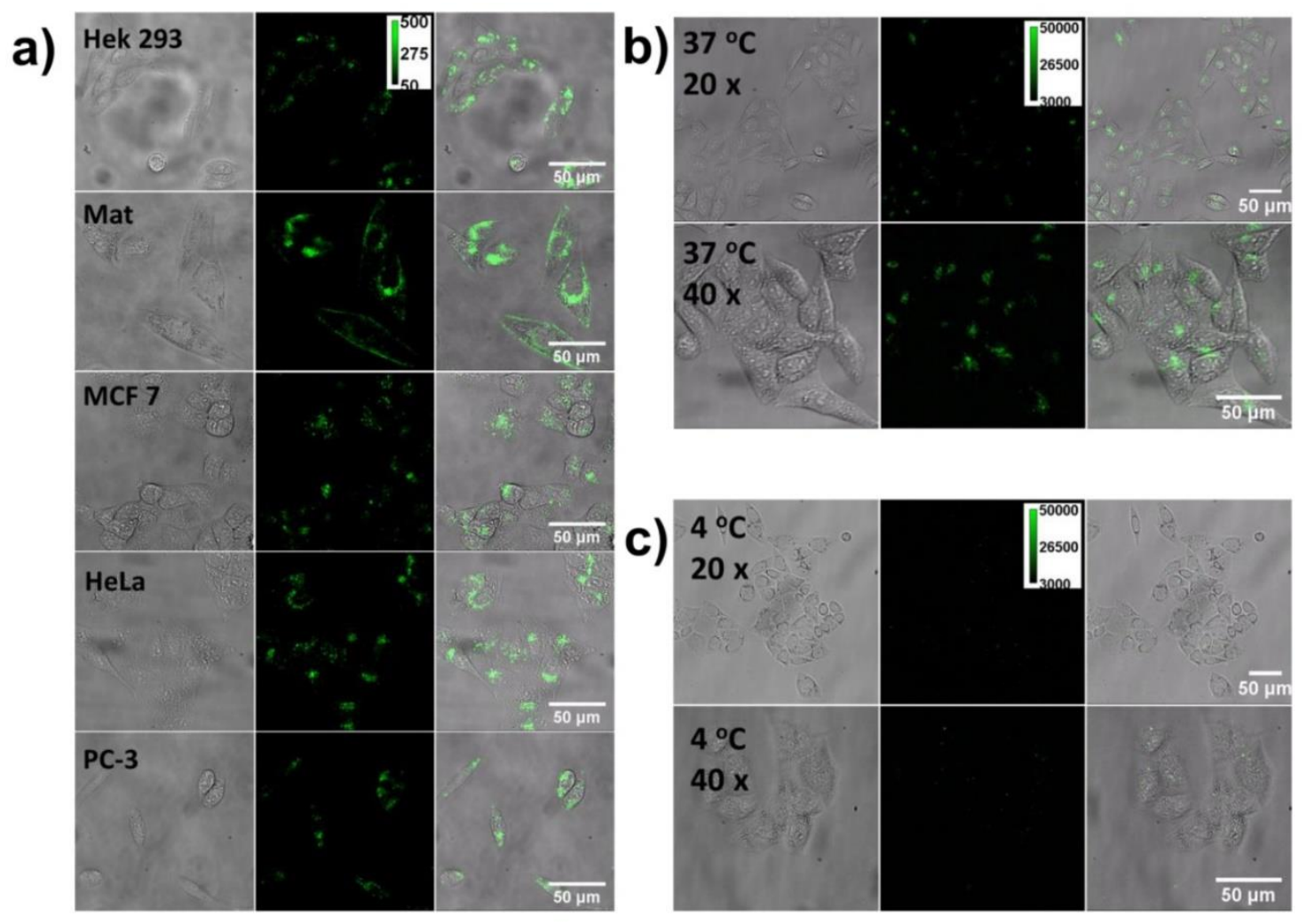

Figure 4. The cellular uptake efficiency assay. a) Five kinds of cells were co-cultured with the CPNPs for $2 \mathrm{~h}$ and then imaged with a fluorescence microscope. Pictures from left to right are the bright-field, fluorescence and merged microscopic images respectively. b) and c) are the fluorescence images of the cells co-cultured with CPNPs for $2 \mathrm{~h}$ at 37 and $4 \circ \mathrm{C}$ respectively. 




Figure 5. The gene expression results after co-cultured the nano-vector with Hek 293 cell for $36 \mathrm{~h}$. i) EGFP+CPNPs, ii) DsRed+CPNPs, iii) EGFP+Lipofectamine 2000, iv) DsRed+Lipofectamine 2000. Pictures from left to right are the bright-field, fluorescence and merged microscopic images respectively.

together in a set of different ratios. The pDNA binding ability toward the CPNPs was then studied by gel electrophoresis assay. As shown in Figure S5, when the ratio (CPNPs/pDNA, w/w) was smaller than 5, evident DNA band (stained with EB) toward the anode was observable after 20 min, indicative of a negatively charged complex moving toward the anode. In other words, under these conditions, the surface charge of the pDNA/CPNPs composites is negatively charged. Normally, a negatively charged surface is not favorable for cell membrane penetration in the absence of recognition antibody because of the electrostatic repulsion barrier formed by the cell membrane.

On this account, we chose the composite with a mass ratio of 5 for the following gene transfection experiment. Figure 5 shows the gene expression results after co-cultured the nano-vector (loaded with pEGFP) with Hek 293 cells for 36 h. Noticeable green fluorescence was observed from the cells with an expression efficiency of $70 \pm 3 \%$. In contrast to the standard control,

\section{Gene Delivery with CPNPs}

Typically, for gene delivery, the nucleic acids need to be coated onto the surface of the nanocargo via chemical or physical association. Herein, through electrostatic interaction, the negatively charged nucleic acid could self-assemble onto the CPNPs surface. Normally, the plasmid DNA (pDNA) to nanovector ratio is an important parameter, which directly determines the membrane penetration efficiency as well as nucleic acid loading efficiency. To achieve high gene transfection efficiency, a balance between these two processes should be established. According to the previous experimental results, the best condition is achieved when the whole surface charge of the vector is neutralized after loading with nucleic acid. From this consideration, we mixed CPNPs with pDNA (by using enhanced green fluorescent protein plasmid as a model, pEGFP) lipofectamine 2000 (55 $\pm 5 \%$ ), the transfection efficiency is noticeably higher under the same transfection condition. It was found that the gene transfection was independent of the type of pDNA applied. Not only for pEGFP, an efficient expression of pDsRed $(65 \pm 5 \%$, a red fluorescent protein plasmid) was also observed by using CPNPs as the vector under the same transfection condition. Furthermore, besides the Hek 293 cells, we also explored the gene delivery capability on HeLa cells. As noted in Figure S6, evident red fluorescence was observed after co-incubated the cell with the composite of CPNPs/pDsRed for $42 \mathrm{~h}$. The excellent gene transfection efficiency as well as non-selectivity toward the type of pDNA enables the promising applicability of these positively charged CPNPs for diverse types of nucleic acid delivery, including gene silence. 


\section{Intracellular Trafficking of CPNPs}

To further understand details about the decent gene transfection efficiency from the CPNPs, we examined the intracellular behavior (i.e. transport, and localization) in living cell with fluorescence microscopy. After co-cultured the cell with the CPNPs for $2 \mathrm{~h}$, evident green fluorescence from the CPNPs in the cytosol appeared, Figure 6, indicative of a successful internalization process. Upon close

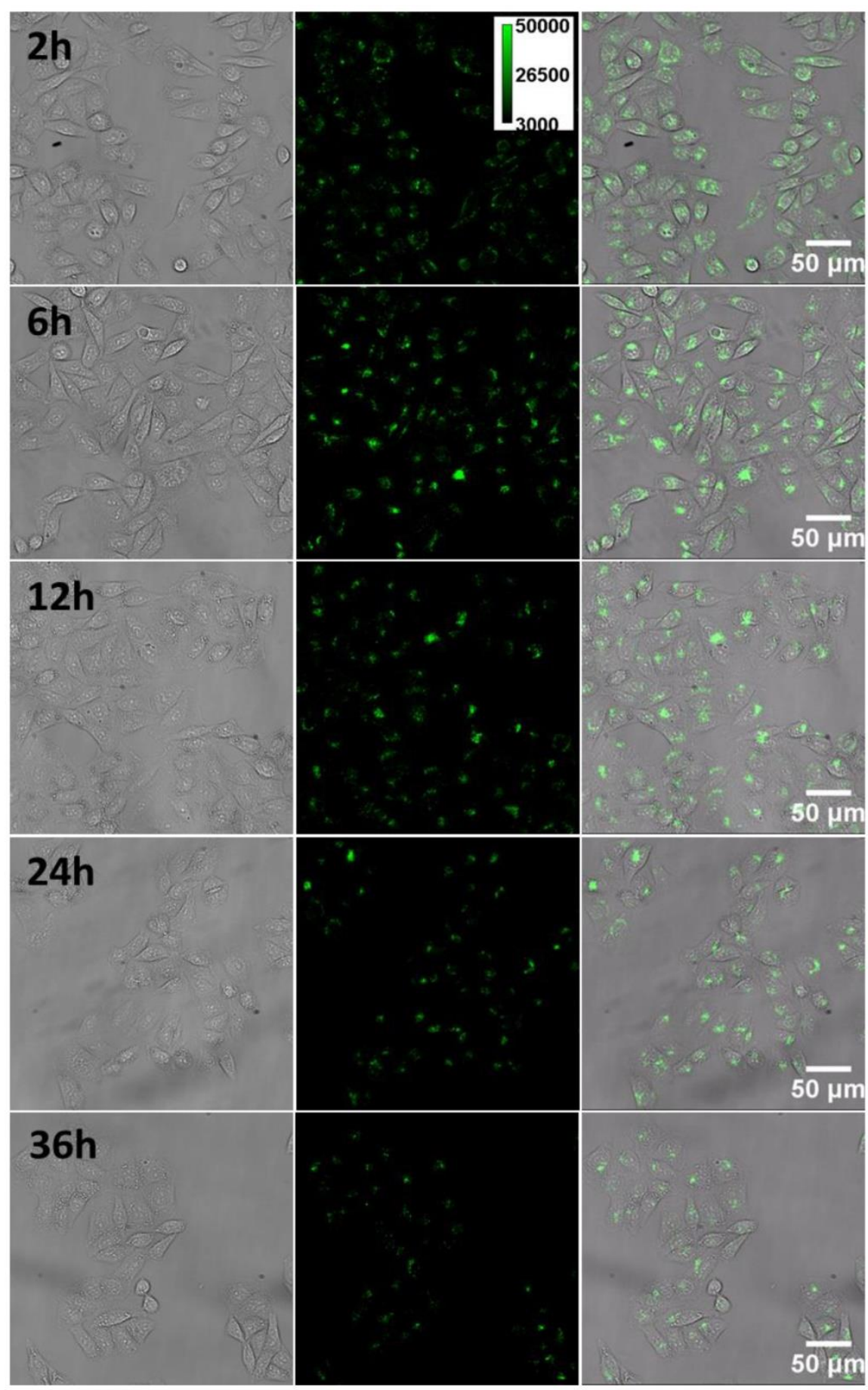

Figure 6. The tempo-spatially resolved microscopic images of CPNPs inside the cell. Pictures from left to right are the bright-field, fluorescence and merged microscopic images of the cells. After internalized by the cell, CPNPs inside the cytosol could gradually assemble close to the periphery of the nucleus as time goes on. inspection of the magnified image, Figure S7, the fluorescence within the cell was not evenly distributed. Individual bright spots randomly dispersed in the cytosol with distinct fluorescence intensity, which is noticeably brighter than individual CPNPs. This observation is in accordance with the above observed results that endocytosis is the major pathway for cellular uptake process. These assembled clusters would be due to the vesicle entrapment effect after the endocytosis process. As time goes on, some of the vesicles fused together and gradually assembled around the nuclei. Similar kind of active motivation was noted in the case of TAT-modified gold nanoparticles [48]. Motor proteins-assisted direct movement plays a key role in the redistribution of CPNPs inside the cell. However, it is worthy of note that, after assembling on the periphery of nuclei, the CPNPs were not dispersed quickly as observed in the case of gold nanoparticles. Even $36 \mathrm{~h}$ later, the majority of CPNPs still located around the nuclei. These observations demonstrated that, during the translation process, the membrane of the vesicles would be broken up. Motor proteins on the cytoskeleton will no more drag the vesicles moving inside the cell actively.

To further confirm this point, we stained lysosome with Lyso-Tracker Red and then imaged the cell with green and red channel simultaneously, Figure 7 . Initially $(4 \mathrm{~h})$, most of the green fluorescent spots could be co-localized with the red color stained lysosomes well. However, when checked the sample at $12 \mathrm{~h}, \quad$ co-localization gradually disappeared, indicative of the escape of 
CPNPs from the vesicle. Normally, endosome escaping is one of the limiting steps for gene delivery. To achieve an efficient gene transfection process, several strategies have been developed based on chemical or physical stimulations such as $\mathrm{pH}$ and temperature [49]. It is worth to point out that, in this case, without introducing external stimuli, the CPNPs could effectively escape from the endosomes. Previous studies have demonstrated that amine group functionalized nanoparticles could break up the vesicle membrane via a proton sponge effect $[25,26]$. In order to show when CPNPs separate from pDNA, we applied a model to mimic this process (the pDNA is not easy to label). That is labeling a positively charged polymer molecules (poly acrylic acid, PAA) with red dyes (Alexa 594-NHS) and then monitor the time-dependent releasing process inside the cell. The zeta potential of this composite was also adjusted to that comparable with the composite of CPNPs/pDNA with the ratio of $5(3.6 \mathrm{mV})$. Initially, the fluorescence signal from the green (CPNPs) and red (dye-labeled pDNA) channels could be overlapped together, Figure S8. Around $24 \mathrm{~h}$ later, some of the green dots were separated from the red dots, indicative of the releasing of the negatively charged polymer from the surface of CPNPs. As a consequence, the good cellular uptake efficiency, time dependent nuclei target capability and the endosome membrane disruption ability together contribute to a high efficiency to translocate pDNA into the cytosol close to the nuclei and subsequently promote the gene expression efficiency.

\section{Conclusion}

In summary, we demonstrated a positively charged small size fluorescent conjugated polymer nanoparticle for efficient gene delivery as well as real time intracellular fluorescence imaging. The CPNPs were made by nanoprecipitation of the conjugated polymer with propylene glycol amine cationic lipid together, resulting in a positively charged $\xi$ potential (i.e., $+46.8 \mathrm{mv}$ for R16). Fast and universal cellular translocation capability was observed according the comprehensive cellular uptake experiments. Nearly $100 \%$ cellular loading efficiency was achieved after co-incubation of the nanoparticle with the cell for $2 \mathrm{~h}$. In comparison with the commonly used gene delivery vector, lipofectamine 2000, the gene expression efficiency was improved significantly. Intracellular fluorescence imaging results demonstrated that the CPNPs could actively assemble close to the periphery of nuclei. Disassembly was not observed even $36 \mathrm{~h}$ later, which greatly facilitates releasing of pDNA close to the periphery of nuclei and thus promotes the gene transfection efficiency. Owing to the merits as described above, the CPNPs demonstrated herein would afford a promising non-viral platform for therapeutic nucleic acids delivery in the future.

\section{Acknowledgements}

This work was supported by National Natural Science Foundation of China (NSFC: 21405045, 21522502), Fundamental Research Funds for the Central Universities: 12060046030 and Distinguished Young Scholar Fund (Hunan province,17B155).
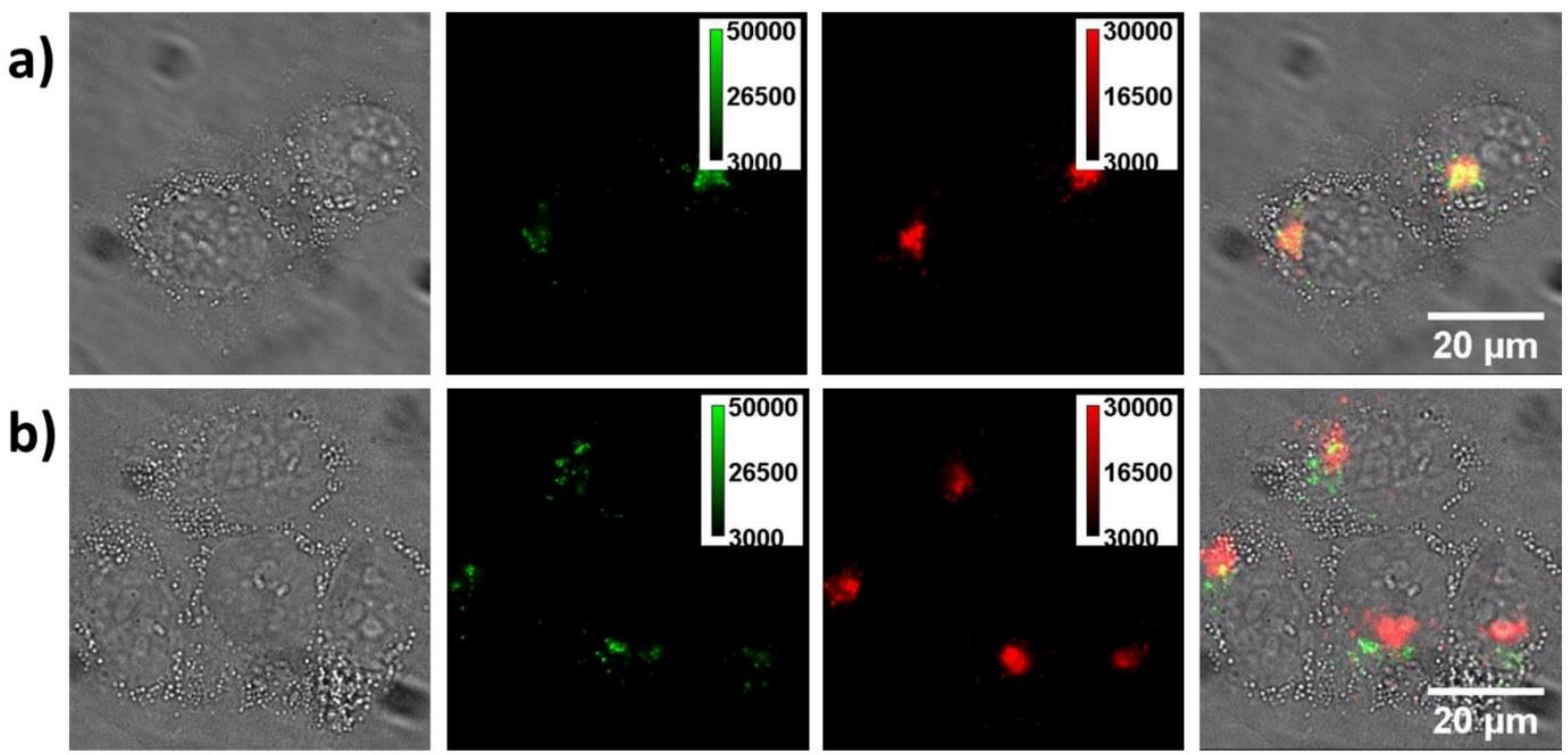

Figure 7. Lysosome staining experiments. a) The cells co-cultured with the CPNPs for $4 \mathrm{~h}$ and then stained with lyso-Tracker Red. Pictures from left to right are the bright-field, green channel fluorescence (from CPNPs), red channel fluorescence (from the lysosome tracker) and merged images of living cells. As noted, the majority of the fluorescent spots from green and red channels are overlapped well, demonstrating that most of the CPNPs are inside the vesicles after internalized by the cell. b) The same staining experiment after co-incubating the cell with CPNPs for $12 \mathrm{~h}$. As demonstrated in the merged image, the fluorescence from the green and red channels cannot be well merged together. However, those signals from the CPNPs are still staying close to the periphery of the nuclei. 


\section{Supplementary Material}

Supplementary figures.

http://www.ntno.org/v02p0157s1.pdf

\section{Competing Interests}

The authors have declared that no competing interest exists.

\section{References}

(1) Feigin A, Kaplitt MG, Tang C, Lin T, Mattis P, Dhawan V, et al. Modulation of metabolic brain networks after subthalamic gene therapy for Parkinson's disease. Proc Natl Acad Sci USA. 2007; 104: 19559-19564.

(2) Kaplitt MG. Parkinson disease: Another player in gene therapy for Parkinson disease. Nat Rev Neurol. 2010; 6: 7-8.

(3) Yang ZR, Wang HF, Zhao J, Peng YY, Wang J, Guinn B-A, et al. Recent developments in the use of adenoviruses and immunotoxins in cancer gene therapy. Cancer Gene Ther. 2007; 14: 599-615.

(4) Mitomo K, Griesenbach U, Inoue M, Somerton L, Meng C, Akiba E, et al. Toward Gene Therapy for Cystic Fibrosis Using a Lentivirus Pseudotyped With Sendai Virus Envelopes. Mol Ther. 2010; 18: 1173-1182.

(5) Verma IM, Weitzman MD. GENE THERAPY: Twenty-First Century Medicine. Annu Rev Biochem. 2005; 74: 711-738.

(6) Morille M, Passirani C, Vonarbourg A, Clavreul A, Benoit J-P. Progress in developing cationic vectors for non-viral systemic gene therapy against cancer. Biomaterials. 2008; 29: 3477-3496.

(7) Li S-D, Huang L. Gene therapy progress and prospects: non-viral gene therapy by systemic delivery. Gene Ther. 2006; 13: 1313-1319.

(8) Thomas CE, Ehrhardt A, Kay MA. Progress and problems with the use of viral vectors for gene therapy. Nat Rev Genet. 2003; 4: 346-358.

(9) Foldvari M, Chen DW, Nafissi N, Calderon D, Narsineni L, Rafiee A. Non-viral gene therapy: Gains and challenges of non-invasive administration methods. J Control Release. 2015; 240: 165-190.

(10) Kauffman KJ, Webber MJ, Anderson DG. Materials for non-viral intracellular delivery of messenger RNA therapeutics. J Control Release. 2016; 240: 227-234.

(11) Li S, Yuan H, Chen H, et al. Cationic Poly (p-phenylene vinylene) Materials as A Multifunctional Platform for Light-Enhanced siRNA Delivery. Chem Asian J. 2016; 11: 2686-2689.

(12) Feng $X$, Tang $Y$, Duan $X$, et al. Lipid-modified conjugated polymer nanoparticles for cell imaging and transfection. J Mater Chem. 2010; 20: 1312-1316.

(13) Yu GS, Choi H, Bae YM, et al. Preparation of cationic polydiacetylene nanovesicles for in vitro gene delivery. J Nanosci Nanotechno. 2008; 8: 5266-5270.

(14) Zhou D, Cutlar L, Gao Y, et al. The transition from linear to highly branched poly( $\beta$-amino ester)s: Branching matters for gene delivery. Sci. Adv. 2016; 2: e1600102-e1600102

(15) Zhou D, Gao Y, Aied A, et al. Highly branched poly( $\beta$-amino ester)s for skin gene therapy. J Control Release. 2016; 244: 336-346

(16) Huang J Y, Gao Y, Cutlar L, et al. Tailoring highly branched poly $(\beta$-amino ester)s: a synthetic platform for epidermal gene therapy. Chem Commun. 2015; 51: 8473-8476.

(17) Salem AK, Searson PC, Leong KW. Multifunctional nanorods for gene delivery. Nat Mater. 2003; 2: 668-671.

(18) Ghosh PS, Kim CK, Han G, Forbes NS. VM Rotello, Efficient Gene Delivery Vectors by Tuning the Surface Charge Density of Amino Acid-Functionalized Gold Nanoparticles. ACS Nano. 2008; 2: 2213-2218.

(19) Guo S, Huang Y, Jiang Q, Sun Y, Deng L, Liang Z, et al. Enhanced Gene Delivery and siRNA Silencing by Gold Nanoparticles Coated with Charge-Reversal Polyelectrolyte. ACS Nano. 2010; 4: 5505-5511.

(20) Liu X, Zhou J, Yu T, Chen C, Cheng Q, Sengupta K, et al. Adaptive Amphiphilic Dendrimer-Based Nanoassemblies as Robust and Versatile siRNA Delivery Systems. Angew Chem Int Ed. 2014; 53: 11822-11827.

(21) Yu T, Liu X, Bolcato-Bellemin A-L, Wang Y, Liu C, Erbacher P, et al. An Amphiphilic Dendrimer for Effective Delivery of Small Interfering RNA and Gene Silencing In Vitro and In Vivo. Angew Chem Int Ed. 2012; 124: 8606-8612.

(22) Zhang Y, Satterlee A, Huang L. In Vivo Gene Delivery by Nonviral Vectors: Overcoming Hurdles? Mol Ther. 2012; 20: 1298-1304.

(23) Guo X, Huang L. Recent Advances in Nonviral Vectors for Gene Delivery. Acc Chem Res. 2012; 45: 971-979.

(24) Mintzer MA, Simanek EE. Nonviral Vectors for Gene Delivery. Chem Rev. 2009; 109: 259-302

(25) Tian W-D, Ma Y-Q. Insights into the endosomal escape mechanism via investigation of dendrimer-membrane interactions. Soft Matter. 2012; 8: 6378-6384.

(26) Yessine M. Membrane-destabilizing polyanions: interaction with lipid bilayers and endosomal escape of biomacromolecules. Adv Drug Deliver Rev. 2004; 56 : 999-1021.
(27) Rosi NL, Giljohann DA, Thaxton CS, Lytton-Jean AKR, Han MS, Mirkin CA. Oligonucleotide-Modified Gold Nanoparticles for Intracellular Gene Regulation. Science. 2006; 312: 1027-1030.

(28) Seferos DS, Prigodich AE, Giljohann DA, Patel PC, Mirkin CA. Polyvalent DNA Nanoparticle Conjugates Stabilize Nucleic Acids. Nano Lett. 2009; 9: 308-311.

(29) Wu C, Schneider T, Zeigler M, Yu J, Schiro PG, Burnham DR, et al Bioconjugation of Ultrabright Semiconducting Polymer Dots for Specific Cellular Targeting. J Am Chem Soc. 2010; 132: 15410-15417.

(30) Chan Y-H, Wu P-J. Semiconducting Polymer Nanoparticles as Fluorescent Probes for Biological Imaging and Sensing. Part Part Syst Charact. 2014; 32: 11-28.

(31) Hashim Z, Howes P, Green M. Luminescent quantum-dot-sized conjugated polymer nanoparticles-nanoparticle formation in a miniemulsion system. J Mater Chem. 2011; 21: 1797-1803.

(32) Howes P, Green M, Levitt J, Suhling K, Hughes M. Phospholipid Encapsulated Semiconducting Polymer Nanoparticles: Their Use in Cell Imaging and Protein Attachment. J Am Chem Soc. 2010; 132: 3989-3996.

(33) Feng L, Zhu C, Yuan H, Liu L, Lv F, Wang S. Conjugated polymer nanoparticles: preparation, properties, functionalization and biological applications. Chem Soc Rev. 2013; 42: 6620-6633.

(34) Tuncel D, Demir HV. Conjugated polymer nanoparticles. Nanoscale. 2010; 2:484.

(35) Barbara PF, Gesquiere AJ, Park S-J, Lee YJ. Single-Molecule Spectroscopy of Conjugated Polymers. Acc Chem Res. 2005; 38: 602-610.

(36) Wei L, Zhou P, Yang Q, Yang Q, Ma M, Chen B, et al. Fabrication of bright and small size semiconducting polymer nanoparticles for cellular labelling and single particle tracking. Nanoscale. 2014; 6: 11351-11358.

(37) Zeigler MB, Sun W, Rong Y, Chiu DT. Hybrid Semiconducting Polymer Nanoparticles as Polarization-Sensitive Fluorescent Probes. J Am Chem Soc. 2013; 135: 11453-11456.

(38) Shimizu KT, Neuhauser RG, Leatherdale CA, Empedocles SA, Woo WK, Bawendi MG. Blinking statistics in single semiconductor nanocrystal quantum dots. Phys Rev B. 2001; 63: 205316.

(39) Kuno M, Fromm DP, Hamann HF, Gallagher A, Nesbitt DJ. Nonexponential "blinking" kinetics of single CdSe quantum dots: A universal power law behavior. J Chem Phys. 2000; 112: 3117-3120.

(40) Geddes CD, Parfenov A, Gryczynski I, Lakowicz JR. Luminescent blinking of gold nanoparticles. Chem Phys Lett. 2003; 380: 269-272.

(41) Xiao L, He Y, Yeung ES. High Throughput Single Molecule Spectral Imaging of Photoactivated Luminescent Silver Clusters on Silver Island Films. J Phys Chem C. 2009; 113: 5991-5997.

(42) Dickson RM, Cubitt AB, Tsien RY, Moerner WE. On/off blinking and switching behaviour of single molecules of green fluorescent protein. Nature. 1997; 388:355-358

(43) Ciapetti G, Cenni E, Pratelli L. A Pizzoferrato, In vitro evaluation of cell/ biomaterial interaction by MTT assay. Biomaterials. 1993; 14: 359-364.

(44) Fotakis G, Timbrell JA. In vitro cytotoxicity assays: Comparison of LDH, neutral red, MTT and protein assay in hepatoma cell lines following exposure to cadmium chloride. Toxicol Lett. 2006; 160: 171-177.

(45) Yin Win K, Feng S-S. Effects of particle size and surface coating on cellular uptake of polymeric nanoparticles for oral delivery of anticancer drugs. Biomaterials. 2005; 26: 2713-2722.

(46) He C, Hu Y, Yin L, Tang C, Yin C. Effects of particle size and surface charge on cellular uptake and biodistribution of polymeric nanoparticles. Biomaterials. 2010; 31: 3657-3666

(47) Chithrani BD, Chan WCW. Elucidating the Mechanism of Cellular Uptake and Removal of Protein-Coated Gold Nanoparticles of Different Sizes and Shapes. Nano Lett. 2007: 7: 1542-1550.

(48) Wei L, Yang Q, Xiao L. Tempo-spatially resolved cellular dynamics of human immunodeficiency virus transacting activator of transcription (Tat) peptide-modified nanocargos in living cells. Nanoscale. 2014; 6: 10207-10215.

(49) Mura S, Nicolas J, Couvreur P. Stimuli-responsive nanocarriers for drug delivery. Nat Mater. 2013; 12: 991-1003. 